\title{
Electricity Monitoring and Interference Reduction of Two-Wire Household Appliances Using Current and Voltage Sensor Tag
}

\author{
Vrushali S. Ajankar ${ }^{1}$, P. N. Pusdekar ${ }^{2}$ \\ ${ }^{1}$ PG Student, Amravati University, P.R.Pote (Patil) Welfare \& Education Trust's College of Engineering\& Management, Maharashtra, India \\ ${ }^{2}$ Assistant Professor, Amravati University, P.R. Pote (Patil) Welfare \& Education Trust's College of Engineering \& Management, \\ Maharashtra, India
}

\begin{abstract}
This review paper demonstrates a flexible nonintrusive current and voltage sensor tag with an interference reduction scheme for accurate electricity monitoring of the household appliances using typical SPT-2 18 AWG zip-cord power lines with wireless analysis using RF modem and ATmegal6 microcontroller. Both current and voltage sensors with the design of a 50-turn inductive coil and two

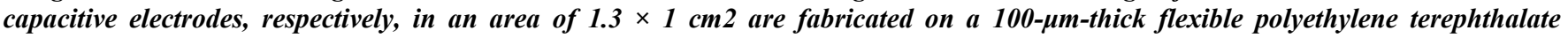
substrate as a sensor tag. The tag exhibits a sensitivity of $271.6 \mathrm{mV} / \mathrm{A}$ and $0.38 \mathrm{mV} / \mathrm{V}$ via active low-pass filter circuits for the current and voltage detection. A compensation circuit inputted with the signals of the voltage sensor is applied for the interference reduction of the current sensor electrically coupled with the power cord so that the current sensor can achieve better signal-to-noise ratio for measuring the loaded current of $1 \mathrm{~A}, 60 \mathrm{~Hz}$ on the power line.
\end{abstract}

Keywords: Flexible sensor and nonintrusive, household electricity monitoring, current and voltage sensor tag, RF module, ATmega16

\section{Introduction}

As the energy deficiency becomes the serious issue today, most of the countries in the world are dedicated to the improvement of the energy efficiency. One of the important energy-reservation strategies is to construct advanced metering infrastructure for the promotion of time-based pricing to reduce peak load, increase the efficiency of energy use, and improve the quality of electricity service. With the concept of "Smart Grids" [1], the electricity monitoring systems possessing the demand response (DR) capability have been reported to be a cost-effective apparatus to well manage electricity usage in residential areas for energy reservation [2].

For the pervasive deployment of the DR monitoring system, robust, low cost, easy to use, and small form factor are the key features of the power sensing system for monitoring $50 / 60-\mathrm{Hz}$ household electricity. A power monitoring system comprises current and voltage sensors. The current sensors have been widely utilized for industrial appliance monitoring applications and are generally designed with the detection of magnetic field generated by the electric current flowing through power cords. Such a current sensor is contactless, very suitable for the pervasive power detection of household appliances owing to nondestructive and easyimplementation characteristics. These current sensors include the Rogowski coil, current transformer (CT), fluxgate, Hall-effect sensor, magnetoimpedance sensor, and so on [3]-[5]. The Rogowski coil is designed with a spiral air-core coil winding around a source conductor for current measurement based on Faraday's law of induction. Without being implemented with a ferromagnetic core, the coil sensor can exhibit highly linear sensitivity and wide operation bandwidth, and it is capable of measuring large current without saturation [6], [7]. For low current measurement, the Rogowski coil must be integrated with a high performance amplifier[8]. Similar to the geometry of the Rogowski coil, CT is designed with the coil structure composed of a primary coil, a secondary coil, and an embedded bulk ferromagnetic ring core [9]. It detects the current by encircling the source conductor with the sensor coils. However, the saturation and hysteresis effects on the magnetization resulting in detection error should be considered while the source current contains a dc component or is loaded with the ac current in a high-frequency regime. Periodical calibration is required for the CT to keep the accuracy in measurement [3]. Since the Rogowski coil and CT can only detect the current flowing through a single current-carrying conductor, they are not useful in the electricity monitoring of household appliances usually accompanied with a power cable containing two or three power wires.

Fluxgate current sensors have two coils, which are driving and sensing coils winding around a ferromagnetic core, respectively, for measuring $\mathrm{dc}$ or low-frequency magnetic fields. Although the resolution can be as low as $10 \mathrm{pT}$, the inevitable large size and intricate reading circuit of the sensor to accomplish precise measurement make the sensor itself not practical for being distributed over household appliances. Nevertheless, large driving current up to 300 $\mathrm{mA}$, i.e., $\sim 100-\mathrm{mW}$ power consumption, or $10-\mathrm{kHz} 600-\mathrm{mA}$ current injecting into the excitation coil is required to ensure the saturation of the core for field sensing. Thus, the necessity of either large power consumption or low temperature operational environment will hinder these sensors for long-term operation, particularly in residential applications.

Magnetoresistivity effect can be utilized for magnetic field detection. The magnetic field-dependent resistance change of conductive materials, such as anisotropic magnetoresistance, giant magnetoresistance (GMR), or tunnel magnetoresistance films, mainly resulted from the spin-orbital coupling effect [12]. The precise thickness and

\section{Volume 5 Issue 1, January 2016}




\section{International Journal of Science and Research (IJSR) \\ ISSN (Online): 2319-7064}

Index Copernicus Value (2013): 6.14 | Impact Factor (2014): 5.611

composition of the multilayered magnetic structures to ensure the magnetized orientation may increase the fabrication cost. The phenomenon of magnetoresistivity change has also been observed in magnetic Permalloy whose resistivity change is caused by the influence of the Lorenz force on the motion of carriers. Although the alloy can be electroplated and utilized as a low-cost magnetic sensor material, nonlinear behavior and large thermal dependence would limit the sensor accuracy.

Hall-effect sensors have been widely applied for dc and ac magnetic field measurement. Via a constant current flowing through a thin conductive layer applied with an external magnetic field, a voltage generated in the perpendicular direction can be utilized as an indicator to evaluate the field strength. In fact, the sensor can be miniaturized using CMOS process to fully integrate a readout circuit for better performance and lower manufacture cost. Recently, Frick et al. presented a silicon Hall current sensor with a differential readout circuit that can detect a $50-\mathrm{Hz}$ current with $0.5 \%$ accuracy in the range of $250 \mathrm{~mA}-5$ A corresponding to a magnetic field of several milliteslas [13]. Nevertheless, while the household appliance is operated, the induced magnetic field around its PVC-insulated power cable is about an order of magnitude smaller than a millitesla that is almost equal to the offset voltage of the CMOS Hall-effect device, i.e., the misalignment voltage at a zero magnetic field. The Hall sensor therefore requires a delicate compensation circuitry for the current detection of household appliances. Although the Hall sensor with higher magnetic sensitivity, lower equivalent magnetic offset, and noise can be achieved by integrating a magnetic flux concentrator that guides the magnetic field in parallel with the chip surface to enhance the detected signal, the concentrator can be useful unless the sensor is not placed near any single wire in the zip cord, i.e., two-wire power line.

On the other hand, contactless voltage sensors are generally designed with the electrical coupling mechanism where sensor electrodes are utilized to collect the charges like a capacitor and connected to a readout circuit. These voltage sensors include the electric-field mill, electro-optical voltage transducer, FET- or varactor-based voltage sensors, etc. [16]-[19]. The sensitivity of the mill depends on the mechanical performance of the shutter requiring a reliable vacuum package for accurate sensing and long-term operation. Noras presented a solid-state electric-field sensor based on variable capacitances capable of detecting 50/60$\mathrm{Hz}$ voltages [19]. The varactors must be driven with a 1$\mathrm{MHz} 10-\mathrm{V}$ signal that requires a complex high voltage circuitry, while they are miniaturized and operated with the button cell battery for household electricity monitoring applications. Although the electro-optical sensor can provide accurate voltage sensing owing to its high immunity to electromagnetic interference, it is usually accompanied by a large, complicated, and expensive signal analyzing system that will make itself impractical for household applications.

Meanwhile, few research works have been presented regarding the electricity monitoring system combining voltage and current sensors together. Kubo et al. showed a voltage/current sensor designed with two air coils and two electrodes for detecting the power transmission along a single power line applied with a kilovolt order of power which has been widely used in industrial manufactories [20]. Rowe et al. presented a nonintrusive load monitoring system by collecting the electromagnetic field generated from a 12 AWG wire using a FET-based electric-field sensor and a coil-based magnetic sensor via a readout circuit [18]. However, these sensing systems are not practical for the proposed power monitoring purpose because either most of the household appliances are equipped with the power input port using a two-wire line or the requirement of the millihertz-order inductor and 5-cm antenna power makes the sensor too large to be pervasively used for household appliances.

Basically, the two-wire power line contains two electric currents flowing in opposite directions, so the magnetic field around each single wire is quite small. The maximum field in the power line is perpendicular to the junction of the two wires with a magnet mounted and placed on the top center of the two-wire power cable to get the largest sensitivity which is $0.1-1.1 \mathrm{mV} / \mathrm{A}$ [14], [15]. In order to realize small form factor, reliable, accurate, and ubiquitous current and voltage detection, we previously demonstrated a nonintrusive, lowcost, and reliable sensor tag closely fitted with the power cords of household appliances for current and voltage detection [21]. Instead of using a micromechanical structure to detect the maximum magnetic field, a planar spiral inductive coil is fabricated on a flexible substrate to sense the current in the two-wire power line of household appliances based on the detection of magnetic flux change. Meanwhile, by taking the advantage of good proximity provided by the flexible tag, capacitive electrodes are also devised on the same tag for voltage sensing. Nevertheless, the current sensor still suffered from an electrical interference problem that resulted from the electric-field coupling of the load on the power cord. Because the proposed power sensor tag can exhibit better design and process integration with the existing CMOS technology and has revealed an alternative low-cost and pervasive currentsensing solution, the detail design, fabrication, characterization, and practice of the tag with an interference reduction scheme will be developed and depicted in this paper for advancing electricity monitoring systems for widespread use.ult in additional noise and will not transmit the side information.

\section{Previous Schemes for Electricity Monitoring}

There are many techniques developed for electricity monitoring in two-wire household appliances some of these are:

1) V. Frick [13]: This paper presents an integrated ac current sensor based on sensitivity-optimized horizontal Hall effect devices and a differential readout chain. This microsystem has been designed for 5-A rms nominal ac current measurement with $5-\mathrm{kV}$ galvanic isolation and $0.5 \%$ accuracy after calibration from $250 \mathrm{~mA}$ to $5 \mathrm{~A}$, over $1.5-\mathrm{kHz}$ bandwidth, which allows up to 30th (25th) harmonic detection in $50-\mathrm{Hz}(60-\mathrm{Hz})$ applications. This qualifies the current sensor microsystem for class 1 products according to international norms for energy meters (IEC44-1 and IEC1036). From the sensing 


\section{International Journal of Science and Research (IJSR) \\ ISSN (Online): 2319-7064}

Index Copernicus Value (2013): 6.14 | Impact Factor (2014): 5.611

element throughout the instrumental chain's output, the signal conditioning is exclusively performed by lownoise standard CMOS analog blocks. Moreover, the whole microsystem features a mixed-signal structure dedicated to auto-balancing. Manufacturing is more complicated.

2) C. A. Schulz [22]: This study presents measurements of the actual worst-case short circuit currents encountered in artificial interlamination shorts. A procedure using an adapted miniaturized Rogowski coil is described. Results show that the maximum short circuit current is not proportional to the core width, but, except for extremely narrow cores, it increases with good approximation linearly with the core width. Peak currents can in large cores reach several amperes even when only two laminations are involved. Not used for two wire household applications.

3) G. Y. Chen [11]: Proof-of-principle experimental demonstration with theoretical modeling is presented for resonantly enhanced Faraday rotation in a microcoil current sensor. The recirculation of resonant light within the coil gives rise to an accumulated polarization rotation and thus improved responsivity. According to simulations, microcoil resonators with sharper resonances could offer significantly larger enhancements. This new type of current sensor has the potential to be ultrafast, compact, and low-cost. Not used for two wire household applications.

4) Y. Ouyang [5]: The giant magnetoresistance (GMR) effect has revolutionized the fields of data storage and magnetic measurement. In this work, a design of a GMR current sensor based on a commercial analog GMR chip for applications in a smart grid is presented and discussed. Static, dynamic and thermal properties of the sensor were characterized. The characterizations showed that in the operation range from 0 to $\pm 5 \mathrm{~A}$, the sensor had a sensitivity of $28 \mathrm{mV} \cdot \mathrm{A}-1$, linearity of $99.97 \%$, maximum deviation of $2.717 \%$, frequency response of $-1.5 \mathrm{~dB}$ at $10 \mathrm{kHz}$ current measurement, and maximum change of the amplitude response of $0.0335 \% \cdot{ }^{\circ} \mathrm{C}-1$ with thermal compensation. In the distributed real-time measurement and monitoring of a smart grid system, the GMR current sensor shows excellent performance and is cost effective, making it suitable for applications such as steady-state and transient-state monitoring. With the advantages of having a high sensitivity, high linearity, small volume, low cost, and simple structure, the GMR current sensor is promising for the measurement and monitoring of smart grids. Not used for two wire household applications.

5) Y. C. Chen [21]: Demonstrates a flexible nonintrusive current and voltage sensor tag with an interference reduction scheme for accurate electricity monitoring of the household appliances using typical SPT-2 18 AWG zip-cord power lines. Both current and voltage sensors with the design of a 50-turn inductive coil and two capacitive electrodes, respectively, in an area of $1.3 \times 1$ $\mathrm{cm} 2$ are fabricated on a $100-\mu \mathrm{m}$-thick flexible polyethylene terephthalate substrate as a sensor tag. The tag exhibits a sensitivity of $271.6 \mathrm{mV} / \mathrm{A}$ and $0.38 \mathrm{mV} / \mathrm{V}$ via active low-pass filter circuits for the current and voltage detection. A compensation circuit inputted with the signals of the voltage sensor is applied for the interference reduction of the current sensor electrically coupled with the power cord so that the current sensor can achieve over a $40-\mathrm{dB}$ signal-to-noise ratio for measuring the loaded current of $1 \mathrm{~A}, 60 \mathrm{~Hz}$ on the power line. Used for two wire household applications.

\section{System Descriptions}

The sensor tag is divided into two subcomponents:

One is current sensor for current sensing, and the other is voltage sensor for voltage detection. Basically, the current sensor is an inductive coil operated by Faraday's law of induction. The voltage sensor comprises two pieces of metal pads working with the capacitive coupling mechanism. Since the magnetic and electric fields generated by the power source have $\mathrm{r}^{-1}$ and $\mathrm{r}^{-2}$ dependence, respectively, where $r$ is the distance from the sensor to the source, the proximity is the key to realize the high sensitivity of the nonintrusive sensors.

\section{Operational Principle of Current Sensor}

As per simulation of the magnetic field generated around a standard SPT-2 18 AWG power cord with a 1-A current input, the largest time-variant magnetic field will exist on the top or bottom sides of the central area of the two-wire cord. Therefore, an inductive coil tag can be used as the current sensor with the largest output voltage signal generated by the electric current flowing through the power cord, while it is closely attached to the power cord on the designated location. According to the Biot-Savart law, the magnetic flux density generated from a single currentcarrying power cord is derived as follows [5][10]:

$$
\overrightarrow{\mathrm{B}}=\frac{\mu 0 \mathrm{I} \cos \omega \mathrm{t}}{2 \pi \mathrm{r}} \hat{\mathrm{a}}_{\phi}(1)
$$

where $\mathrm{B}$ is the magnetic flux density, $\mathrm{I}$ is the current inside the cord, $\omega$ is the angular frequency of current, and $r$ is the distance from the source to a point in space. Thus, according to Faraday's law of induction, the open-loop current-sensing coil's induced voltage, which is proportional to the magnetic flux change resulting from the time-variant current flowing through the power cord, can be calculated as follows:

$$
V_{i n}=-\sum_{n=1}^{N} \frac{d \phi_{n}}{d t}=-\sum_{n=1}^{N} \frac{d}{d t} \oint \frac{\cos w t \mu_{0}}{\pi t} I \hat{a}_{\phi} \cdot d \widehat{A}_{n}(2)
$$

where $V_{\text {in }}$ is the induced voltage of the coil, $\mathrm{N}$ is the total number of the coil, $A_{n}$ is the area of the nth turn of the coil, and $\phi_{n}$ is the integral of the vertical component of magnetic flux passing through the nth turn area. The sensitivity depends on the dimension of the power cord, the thickness of the flexible substrate, the linewidth, spacing, turns of the sensing coil, etc. In this paper, the sensing coil is having 50 turns designed with spacing of $21 \mu \mathrm{m}$ and linewidth of 21 $\mu \mathrm{m}$ and in an area of $10 \times 5 \mathrm{~mm}^{2}$.

\section{Operational Principle of Voltage Sensor}

Distribution of the electric field in a two wire household power cord, with the neutral and fire lines applied with 0 and $115 \mathrm{~V}$ are located on the left and right sides of the cord, respectively. The electric field normal to the half circle interface on the right-hand side of the fire line is the best location with a sensing electrode that can exhibit the densest electrical flux, i.e., largest induced charge density, than that 


\section{International Journal of Science and Research (IJSR) \\ ISSN (Online): 2319-7064}

Index Copernicus Value (2013): 6.14 | Impact Factor (2014): 5.611

in any other location surrounding the power cord. Therefore, once the proposed voltage sensor comprising two electrodes pinches a power cord to sense a sinusoidal electric field in the power cord, an ac current can be induced between the electrode and the inner conductor of the fire line

$$
\begin{aligned}
& \mathrm{i}_{\mathrm{C}}=\mathrm{C} \frac{d v_{c}}{d t} \\
& \mathrm{~V}_{\mathrm{M}}=\mathrm{i}_{\mathrm{C}} \mathrm{R}_{\mathrm{L}}
\end{aligned}
$$

where $\mathrm{C}$ and $\mathrm{V}_{\mathrm{c}}$ are the capacitance and the voltage difference between the electrode and the inner conductor of the fire line, respectively. The induced current is equal to the multiple of the time derivative of the induced voltage and the capacitance proportional to the electrode area. The output voltage will depend on the total resistance of the connected circuit loop. To determine the characteristics of the voltage sensor, the capacitance between the cylindrical electrode and the inner conductor of the fire line, can be estimated as follows:

$$
\mathrm{C}_{\mathrm{L}}=\left[\sum_{n=1}^{N} \frac{\ln \left(r_{n+1} / r_{n}\right)}{\varepsilon_{r, n} \varepsilon_{0} \theta}\right]^{-1}
$$

where $C_{L}$ is the partial coaxial capacitance per unit length, $\mathrm{r}_{\mathrm{n}}$ is the radius from the fire line center to the interface of the $n$th dielectric, i.e., PVC and polyethylene terephthalate (PET), $\varepsilon_{r, n}$ is the relative permittivity of the $n_{\text {th }}$ dielectric, $\varepsilon_{0}$ is the vacuum permittivity, and $\theta$ is the angle that the sensing electrode covered.

An alternative method is employed to estimate the fringing capacitance as the fringing effect must be considered in this case since the ratio of the perimeter to the area in the sensing electrode cannot be neglected in the micrometer regime here the capacitor is treat as a parallel-plate capacitor as follows

$$
\mathrm{C}_{\mathrm{F}} \cong \varepsilon_{r, 1} \mid \varepsilon_{0}\left[\frac{\omega}{h} \frac{h}{\pi \omega}+\frac{h}{\pi \omega}\left(\frac{2 \pi \omega}{h}\right)\right]
$$

where $\mathrm{C}_{\mathrm{F}}$ is the fringing capacitance part per unit length, $\omega$ is the width of the sensing electrode, and $h$ is the gap distance between the electrode and the inner conductor surface. While the induced voltage on the sensing electrode, $\mathrm{V} 1$, that is connected to a resistance load is much smaller than the voltage on the inner conductor of the fire line, $V_{\text {in }}$, the ac current in (3) can be derived as follows:

$$
\mathrm{i}_{\mathrm{c}}=\mathrm{c} \frac{d v_{c}}{d t}=\mathrm{c} \frac{d\left(v_{\text {in }}-v_{1}\right)}{d t} \approx \mathrm{c} \frac{d v_{\text {in }}}{d t} \text { while } v_{\text {in }} \gg v_{1}
$$

By introducing the current $i_{c}$ to the readout circuit, the output voltage can be therefore estimated. The voltage sensor is used for two purposes first for measuring the electricity voltage of the power cord and second for eliminating the interference noise of the current sensor.

\section{Device Fabrication}

Flexibility for close contact is a required design feature in the proposed sensor Since the proximity is critical to the sensor performance, and it can be realized by the developed technology to fabricate inductive coil and sensing electrodes on a flexible substrate simultaneously. In this paper, a flexible PET substrate instead of SU-8 glued to a silicon wafer is utilized for the flexible power sensor fabrication. The PET can have less residual stress and the same capability as SU-8 for flip-chip bonding with CMOS chips at a temperature below $250{ }^{\circ} \mathrm{C}$ at wafer level[4]. In addition, the PET is transparent and mechanically strong and has good resistance to moisture and chemicals. It is also inexpensive and can be easily acquired.

The fabrication process includes the following:

1) PET substrate preparation;

2) $\mathrm{Ti} / \mathrm{Cu}(30 \mathrm{~nm} / 90 \mathrm{~nm})$ adhesion seed layer deposition;

3 ) inductor and capacitive electrode fabrication; and

4) sensor tag realization.

PET substrate having a $100-\mu$ m-thick A4 size slide, cutting to form a circular PET film which is glued onto a 4-in silicon handling wafer. The wafer is then sputtered with a layer of $\mathrm{Ti} / \mathrm{Cu}(30 \mathrm{~nm} / 90 \mathrm{~nm})$ as an adhesion/seed layer. The inductive coil area of the current sensor and the electrodes area of the voltage sensor on the wafer are defined by AZ4620 photoresist using the conventional photolithography technique and then electroplated with a $2-\mu$ m-thick $\mathrm{Cu}$ coil and capacitor electrodes. After the removal of the AZ4620 photoresist by acetone and the $\mathrm{Ti} / \mathrm{Cu}$ seed/adhesion layer by buffered $\mathrm{HF}$ and $\mathrm{Cu}$ etchant, respectively, the $\mathrm{Cu}$ surface is electroless plated with a thin $\mathrm{Ni} / \mathrm{Au}$ layer for protecting the coil and electrodes from oxidation and corrosion. The metal patterns are covered with a $2-\mu$ m-thick SU-8 layer left with an opening via hole for air-bridging connection by photolithography. Another $\mathrm{Ti} / \mathrm{Cu}$ adhesion/seed layer is sputtered and covered with a photopatterned AZ4620 photoresist as the mold for electroplating $\mathrm{Cu}$ bridge. After electroplating $\mathrm{Cu}$, the $\mathrm{AZ4620}$ photoresist and $\mathrm{Ti} / \mathrm{Cu}$ layer are removed using acetone and buffered $\mathrm{HF}$ and $\mathrm{Cu}$ etchant, respectively. After detaching the PET substrate from the Si handling wafer, the

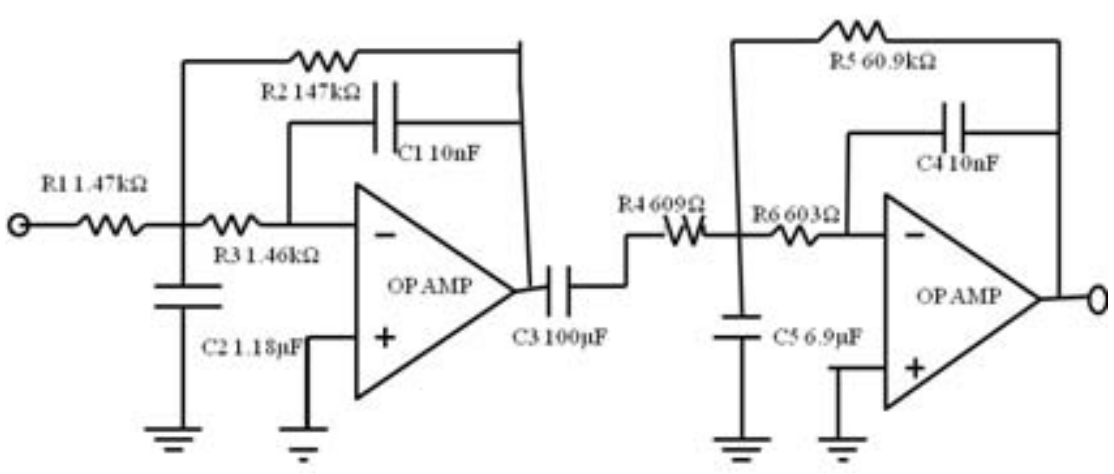

(Gain $=79.5 \mathrm{db}$ in measurement)

(a) 


\section{International Journal of Science and Research (IJSR) \\ ISSN (Online): 2319-7064}

Index Copernicus Value (2013): 6.14 | Impact Factor (2014): 5.611

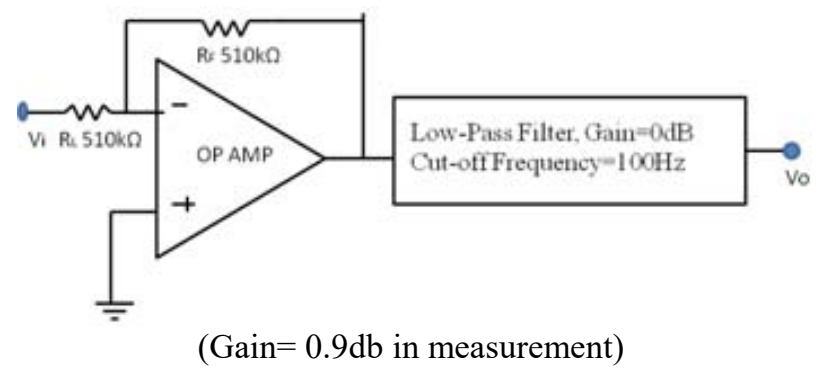

(b)

Fig. 1. (a) Active filter circuit with $80-\mathrm{dB} 100-\mathrm{Hz}$ cutoff frequency designed by TI FilterPro for the current sensor and (b) filter circuit designed for the voltage sensor with 0 $\mathrm{dB} 100-\mathrm{Hz}$ cutoff frequency. The inverter is connected to the voltage sensor for providing a current path to virtual ground. sensor tag is accomplished by cutting the whole PET into pieces of the tags. Using fourth order Butterworth low-pass filter functioning as a low-pass active filter to in reduction circuitry will give better results.

\section{RF Modem and ATmega16}

RF modem is used for applications so set up wireless communication and analyze in matlab will get better result than previous. RF modem features adjustable data rate and reliable transmission distance. The communication protocol is self controlled and completely transparent to user interface. The ATmegal6 is a low-power CMOS 8-bit microcontroller based on the AVR enhanced RISC architecture. By executing powerful instructions in a single clock cycle, the ATmega16 achieves throughputs approaching 1 MIPS per MHz allowing the system designer to optimize power consumption versus processing speed.

\section{Conclusion}

In this paper, a review is presented on various methods for electricity monitoring and efficient use a flexible current and voltage sensor tag. The tag comprises current and voltage sensors fabricated on the same flexible PET substrate using a flexible wafer-level chip-scale fabrication process and operated by Faraday's induction law and capacitive coupling principle, respectively. An interference reduction scheme will be studied. The practicality of the proposed system will be studied and proved with analysis using RF module and ATmega16.

\section{References}

[1] J. Scheffran and A. Battaglini, "Climate and conflicts: The security risks of global warming," Reg. Environ. Change, vol. 11, no. 1, pp. 27-39, Mar. 2011.

[2] E. A. Arens, D. Auslander, D. Culler, C. Federspiel, C. Huizenga, J. Rabaey, P. Wright, and D. White, Demand Response Enabling Technology Development, Phase I Report, Berkeley, CA, USA. [Online]. Available: http://www.escholarship.org/uc/item/0971h43j

[3] P. Ripka, "Electric current sensor: A review," Meas. Sci. Technol., vol. 21, no. 11, pp. 112001-1-112001-23, Nov. 2010
[4] F. S. Shieu, C. F. Chen, J. G. Sheen, and Z. C. Chang, "Intermetallic phase formation and shear strength of a Au-In microjoint," Thin Solid Films, vol. 346, no. 1/2, pp. 125-129, Jun. 1999. [5] C. Xiao, "An overview of integratable current sensor technologies," in Conf. Rec. IEEE 38th IAS Annu. Meeting, Salt Lake City, UT, USA, Oct. 2003, vol. 2, pp. 1251-1258.

[5] Y. Ouyang, J. He, J. Hu, and S. X. Wang, "A current sensor based on the giant magnetoresistance effect: Design and potential smart grid applications," Sensors, vol. 12, no. 11, pp. 15 520-15 541, Nov. 2012

[6] A. Radun, "An alternative low-cost current-sensing scheme for highcurrent power electronics circuits," IEEE Trans. Ind. Electron., vol. 42, no. 1, pp. 78-84, Feb. 1995.

[7] E. Abdi-Jalebi and R. McMahon, "High-performance low-cost Rogowski transducers and accompanying circuitry," IEEE Trans. Instrum. Meas., vol. 56, no. 3, pp. 753-759, Jun. 2007.

[8] P. Poulichet, F. Costa, and E. Laboure, "A new highcurrent largebandwidthdc active current probe for power electronics measurements," IEEE Trans. Ind. Electron., vol. 52, no. 1, pp. 243-254, Feb. 2005.

[9] D. K. Cheng, Field and Wave Electromagnetics, 2nd ed. Reading, MA, USA: Addison-Wesley, 1989.

[10] G. Y. Chen, T. Lee, R. Ismaeel, G. Brambilla, and T. P. Newson, "Resonantly enhanced Faraday rotation in an microcoil current sensor," IEEE Photon. Technol. Lett., vol. 24, no. 10, pp. 860-862, May 2012.

[11]N. A. Stutzke, S. E. Russek, D. P. Pappas, and M. Tondra, "Lowfrequency noise measurements on commercial magnetoresistive magnetic field sensors," $J$. Appl. Phys., vol. 97, no. 10, pp. 10Q107-1-10Q107-3, May 2005.

[12] V. Frick, L. Hébrard, P. Poure, F. Anstotz, and F. Braun, "CMOSmicrosystem for ac current measurement with galvanic isolation," IEEE Sensors J., vol. 3, no. 6, pp. 752-760, Dec. 2003.

[13]E. S. Leland, P. K. Wright, and R. M. White, “A MEMS ac current sensor for residential and commercial electricity end-use monitoring," J. Micromech. Microeng., vol. 19, no. 9, pp. 094018-1-094018-6, Sep. 2009.

[14]E. S. Leland, C. T. Sherman, P. Minor, R. M. White, and P. K. Wright, "A new MEMS sensor for ac electric current," in Proc. IEEE Sensors, Waikoloa, HI, USA, Nov. 2010, pp. 1177-1182.

[15] G. Wijeweera, B. Bahreyni, C. Shafai, A. Rajapakse, and D. R. Swatek, "Micromachined electric-field sensor to measure ac and dc fields in power systems," IEEE Trans. Power Del., vol. 24, no. 3, pp. 988-995, Jul. 2009.

[16] Texas Instruments, FilterProTM. [Online]. Available: http://www.ti.com/ tool/filterpro

[17]A. Rowe, M. Berges, and R. Rajkumar, "Contactless sensing of appliance state transitions through variations in electromagnetic fields," in Proc. ACM BuildSys., Zurich, Switzerland, Nov. 2010, pp. 19-24.

[18] M. A. Noras, "Solid state electric field sensor," in Proc. ESA Annu. Meet. Electrostat., Cleveland, OH, USA, Jun. 2011, pp. 1-6.

[19] T. Kubo, T. Furukawa, H. Fukumoto, and M. Ohchi, "Numerical estimation of characteristics of voltage- 


\section{International Journal of Science and Research (IJSR) \\ ISSN (Online): 2319-7064}

Index Copernicus Value (2013): 6.14 | Impact Factor (2014): 5.611

current sensor of resin molded type for $22 \mathrm{kV}$ power distribution systems," in Proc. ICCAS-SICE, Fukuoka, Saga, Japan, Aug. 2009, pp. 5050-5054.

[20] Y. C. Chen, W. H. Hsu, S. H. Cheng, and Y. T. Cheng, "A flexible, non-intrusive power sensor tag for the electricity monitoring of two-wire household appliances," in Proc. IEEE Int. Conf. MEMS, Paris, France, Jan. 2012, pp. 620-623.

[21]C. A. Schulz, S. Duchesne, D. Roger, and J.-N. Vincent, "Short circuit current measurements between transformer sheets," IEEE Trans. Magn., vol. 46, no. 2, pp. 536-539, Feb. 2010. 